\title{
Gap perception in bumblebees
}

\author{
Sridhar Ravi ${ }^{1,2, *}$, Olivier Bertrand ${ }^{1}$, Tim Siesenop ${ }^{1}$, Lea-Sophie Manz ${ }^{1,3}$, Charlotte Doussot ${ }^{1}$, Alex Fisher ${ }^{2}$ and \\ Martin Egelhaaf ${ }^{1}$
}

\begin{abstract}
A number of insects fly over long distances below the natural canopy, where the physical environment is highly cluttered consisting of obstacles of varying shape, size and texture. While navigating within such environments, animals need to perceive and disambiguate environmental features that might obstruct their flight. The most elemental aspect of aerial navigation through such environments is gap identification and 'passability' evaluation. We used bumblebees to seek insights into the mechanisms used for gap identification when confronted with an obstacle in their flight path and behavioral compensations employed to assess gap properties. Initially, bumblebee foragers were trained to fly though an unobstructed flight tunnel that led to a foraging chamber. After the bees were familiar with this situation, we placed a wall containing a gap that unexpectedly obstructed the flight path on a return trip to the hive. The flight trajectories of the bees as they approached the obstacle wall and traversed the gap were analyzed in order to evaluate their behavior as a function of the distance between the gap and a background wall that was placed behind the gap. Bumblebees initially decelerated when confronted with an unexpected obstacle. Deceleration was first noticed when the obstacle subtended around $35 \mathrm{deg}$ on the retina but also depended on the properties of the gap. Subsequently, the bees gradually traded off their longitudinal velocity to lateral velocity and approached the gap with increasing lateral displacement and lateral velocity. Bumblebees shaped their flight trajectory depending on the salience of the gap, indicated in our case by the optic flow contrast between the region within the gap and on the obstacle, which decreased with decreasing distance between the gap and the background wall. As the optic flow contrast decreased, the bees spent an increasing amount of time moving laterally across the obstacles. During these repeated lateral maneuvers, the bees are probably assessing gap geometry and passability.
\end{abstract}

KEY WORDS: Sensorimotor system, Cluttered environment, Flight, Optic flow, Vision

\section{INTRODUCTION}

Even with relatively tiny brains, insects display a rich repertoire of behaviors, the operation of many of which remains unclear. Aerial locomotion below the natural canopy is one such behavior that has received increased attention in the last decade from biologists and engineers alike (Shyy et al., 2016). Natural flight at the small scale

\footnotetext{
${ }^{1}$ Department of Neurobiology and Cluster of Excellence Cognitive Interaction Technology (CITEC), Bielefeld University, 33615 Bielefeld, Germany. ${ }^{2}$ School of Engineering, RMIT University, Melbourne, VIC 3001, Australia. ${ }^{3}$ Faculty of Biology, Johannes Gutenberg-Universität Mainz, 55122 Mainz, Germany.

*Author for correspondence (Sridhar.ravi@uni-bielefeld.de)

(D) S.R., 0000-0001-7397-9713
}

Received 5 May 2018; Accepted 26 October 2018 of insects, where the sensory and mechanical constraints are particularly challenging, requires the concerted coordination of their computationally parsimonious sensorimotor system (Dudley, 2002). The spatial environment close to the Earth's surface consists of a myriad of natural and artificial objects that can vary widely in shape, size and texture. This renders the physical environment unpredictable and poses challenges to aerial locomotion. Steady level flight for sustained durations is generally unfeasible in this domain, with obstacles constantly getting in the way. Thus, in order to achieve safe transit through such environments, flying systems need to be adept at perceiving the environment to identify obstacles and devise alternative flight paths. From a biophysical standpoint, apart from performance limitations based on allometric body size scaling, other factors such as collision avoidance and properties of the physical environment also influence flight trajectories and the overall performance of insects (Crall et al., 2015; Dudley, 2002).

Unlike during legged locomotion where tactile sensory inputs can augment vision in gaining environmental information, flying insects rely only on vision for safe passage and path planning. For long-distance navigation, flying insects might use other sensory modalities, apart from vision, such as odor and geomagnetic fields (Knaden and Graham, 2016). In order for a flying animal to arrive at its intended destination or ensure safe locomotion, at a basic level, the animal needs to process the obstacles that lie in its path and identify gaps. Obstacle and gap detection may thus be considered the most basic element of flight through clutter. A few recent studies have analyzed the response of flying insects in minimally cluttered environments and revealed that, when confronted with obstacles with varying spacing, insects such as bumblebees and honeybees choose the larger gap (Baird and Dacke, 2016; Ong et al., 2017). This might seem as an obvious response, yet it highlights the active response of insects in avoiding collisions, which otherwise can result in irreparable damage to body and wings. Baird and Dacke (2016) suggested bumblebees may utilize a simple brightness-based strategy in making a choice among the different gaps, i.e. bigger gaps are likely to be brighter than smaller gaps. Although a few experiments have observed insect behavior around individual obstacles and minimally cluttered environments, the mechanisms mediating the elemental process of obstacle and gap perception and the factors that influence the assessment of passability are still unclear.

In particular, fast flying animals, such as many insect species, rely on optic flow as the main source of spatial information, i.e. on the continuous stream of retinal image changes induced during selfmotion, and thus this is particularly relevant for behavior in cluttered environments (Egelhaaf et al., 2010; Egelhaaf et al., 2012). Optic flow has also been shown to aid in estimating flight distance, flight path centering, identification of foraging locations and many other behaviorally relevant tasks (see Baird et al., 2013; Kern et al., 2012; Serres and Ruffier, 2017; Serres et al., 2008; Srinivasan, 2015; Srinivasan and Zhang, 1997). Observation of the flight 
trajectory of insects such as flies and bees has shown that insects actively shape the temporal structure of their visual input by employing prototypical flight maneuvers, especially to separate translational from rotational optic flow and, thus, to facilitate discerning spatial information about the surroundings (Braun et al., 2010, 2012; Egelhaaf et al., 2012). As only translational optic flow contains spatial information, active flight and gaze strategies are believed to facilitate spatial vision and thus may be particularly relevant for navigation in cluttered terrain (Egelhaaf et al., 2014). However, this vision-based strategy relying on the closed action perception loop has not been investigated systematically in cluttered environments and thus needs further investigation. Specifically, how does an insect react to unexpected obstacles obstructing its flight path? What flight maneuvers does it perform in order to detect gaps and assess passability?

Here, we sought to uncover the mechanisms implemented by flying insects in gap identification and perception. Bumblebees are excellent model organisms because much is known about their flight and navigational performance (Baird and Dacke, 2012; Crall et al., 2014; Mirwan and Kevan, 2013; Osborne et al., 2008; Ravi et al., 2013; Riabinina et al., 2014; Lobecke et al., 2018). We presented unsuspecting bumblebees with an altered environment consisting of a wall obstructing their flight path but containing a gap, preventing direct passage to their goal, and observed their behavior as they approached and traversed the gap. We analyzed the flight trajectory of the bees at different distances from the gap and computed key visual metrics such as the angle subtended by the obstacle and gap on the retina, mean optic flow and optic flow contrast in order to identify factors that influence gap identification and assessment of passability. Our data suggest that bumblebees employ an active gazing flight strategy in enabling the identification of gaps and critical environmental parameters that affect safe passage.

\section{MATERIALS AND METHODS Experimental setup}

Experiments were conducted with individuals from a Bombus terrestris (Linnaeus 1758) colony that was maintained within the lab. A healthy hive sourced from a commercial breeder (Koppert Biological Systems) was placed within a $0.5 \times 0.5 \times 0.3 \mathrm{~m}$ mesh enclosure that was covered with dark cloth to simulate the natural underground habitat of the bees. The hive enclosure was connected to a flight tunnel $(0.25 \times 0.25 \times 1.5 \mathrm{~m})$ that led to a $1 \times 1 \times 0.75 \mathrm{~m}$ foraging chamber where gravity feeders containing $30 \% \mathrm{v} / \mathrm{v}$ sucrose solution blended with $1 \%$ commercial honey were placed. Connections between the hive enclosure, flight tunnel and foraging chamber were made using $30 \mathrm{~mm}$ i.d. and $150 \mathrm{~mm}$ long flexible silicon tubing. Finely ground pollen was placed directly within the hive and bees were permitted to access sucrose in the foraging chamber ad libitum. Consistent foraging flights by numerous $(>20)$ worker bees were observed within 1 day of movement of the hive to the enclosure. The temperature within the hive enclosure, flight tunnel and foraging chamber was maintained at $23^{\circ} \mathrm{C}$. Ample natural lighting from the windows was available for the bees. The bees and hive were given 1 week for habituation to the environment before experiments began. During the experiments, blinds over the windows were drawn and a large diffuser was placed in front of a $1000 \mathrm{~W}$ halogen floodlight to create nominally homogeneous lighting. The bees were given around $30 \mathrm{~min}$ to become habituated to the lighting and no difference in the bees' behavior was noted under artificial lighting. The experiments were conducted over 7 consecutive days and blinds were removed upon completion of each experimental bout.
During experiments, gates on either side of the flight tunnel were used to regulate traffic, and only one bee at a time was permitted to enter the flight tunnel. Only bees returning to the hive were considered for analysis. The experiment procedure will be described from the perspective of the bee returning to the hive as per Fig. 1A. An obstacle was created within the flight tunnel by the addition of an artificial vertical wall that contained a $50 \mathrm{~mm}$ wide rectangular hole starting from the middle and extending to the top (see Fig. 1A). The sidewalls of the tunnel were lined with an achromatic random checkerboard pattern (see below) while the floor was lined with a random cloud with spatial frequencies varying by $1 / f$, similar to that used by Monteagudo et al. (2017). A second vertical wall was placed behind the wall containing the gap. Both obstructing walls were covered in the checkerboard pattern (Fig. 1), which consisted of randomly distributed $6 \mathrm{~mm}$ black and white squares. Five different experimental conditions were tested where the distance between the gap and rear wall was set to 550, 300, 150, 50 or $0 \mathrm{~mm}$. During the different scenarios, the wall containing the gap was always placed $0.9 \mathrm{~m}$ from the entrance of the tunnel (see Fig. 1A). Twenty flights were recorded for each condition and the conditions were varied pseudo-randomly between each recording. Once the bees had approached and passed the gap, the rear wall was removed to permit their onward flight back to the hive. For the condition when the rear wall was adjacent to the gap $(d=0 \mathrm{~mm})$, passage was obviously impossible; once $20 \mathrm{~s}$ of recording was completed, the wall with the gap and the rear wall were both removed by opening the roof of the flight tunnel. We also observed the flight of the bees when the wall behind the gap was lined with non-textured white paper and placed immediately adjacent to the gap, i.e. similar to the $d=0 \mathrm{~mm}$ condition (Fig. 1).

To ensure we captured the response of naive bees dealing with a complex environment and negotiating a gap, experiment bouts lasted no longer than $1 \mathrm{~h}$, and the gap and rear wall were removed after each flight recording to inhibit the bees from becoming familiar with the experiment paradigm. Bees were not individually marked in this study; although this increased the possibility of taking unequal numbers of measurements among the different individuals for each condition, the likelihood was greatly reduced as consecutive flights were taken from different bees returning to the hive from the foraging arena. Additionally, because of the large number of foragers and flight trajectories recorded, our dataset is likely to be representative of the population. All experiments were completed within 5 consecutive days.

\section{Flight trajectory analysis and optic flow estimation}

An Optronis CR6 high-speed camera was placed $1.7 \mathrm{~m}$ above the midline of the flight tunnel looking directly downward. The flights of the bees were recorded at $200 \mathrm{~Hz}$, and a region covering $950 \mathrm{~mm}$ leading to the gap was kept in the field of view. The ceiling of the flight tunnel consisted of $5 \mathrm{~mm} U \mathrm{UV}$-permitting transparent acrylic panels that spanned the width of the tunnel. The majority of bees never collided with the roof while on foraging bouts and only such flights were considered for analysis here. During post-processing, lens distortion was corrected by using standard MATLAB Image Processing Toolbox routines. An object of known dimensions was placed within the field of view at mid-height of the tunnel and related to the pixels in the rectified image for 2D spatial calibration. Custom MATLAB code was written to process each frame and fit an ellipse to the body of the bees; subsequently, the centroid location, body length and heading were all measured over the entire flight. The bees displayed a wide diversity of flight behaviors. In the flight tunnel, flights ranged from appearing to explore the space to making 


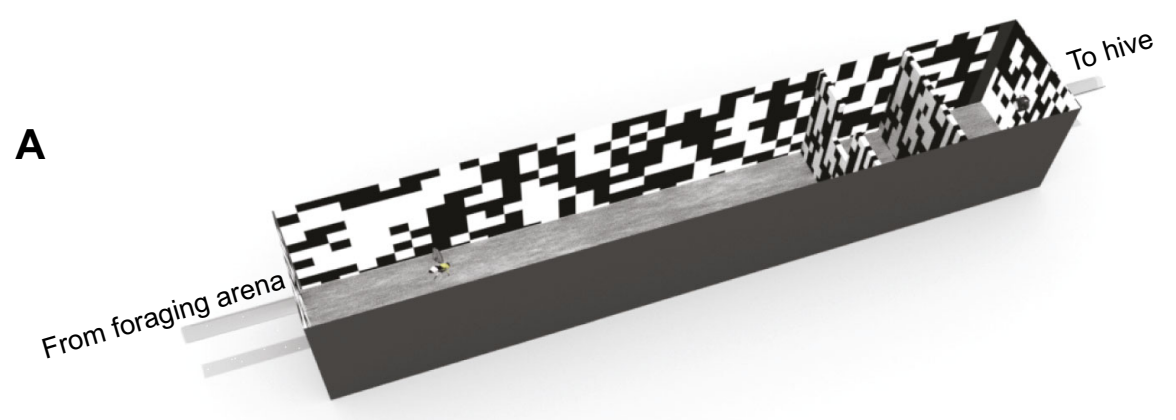

Fig. 1. Experimental setup. (A) Schematic diagram of the setup: $d$ represents the distance between the wall containing the gap and the rear wall. Only the flight of bees returning to the hive from the foraging arena was considered for analysis. (B-F) Sample flight trajectory of a bee when the distance between the gap and rear wall was $550 \mathrm{~mm}(\mathrm{~B})$, $300 \mathrm{~mm}(\mathrm{C}), 150 \mathrm{~mm}(\mathrm{D}), 60 \mathrm{~mm}(\mathrm{E})$ and $0 \mathrm{~mm}(\mathrm{~F})$. The representation of the gap and rear wall on the right is for illustrative purposes only and not to scale.

B

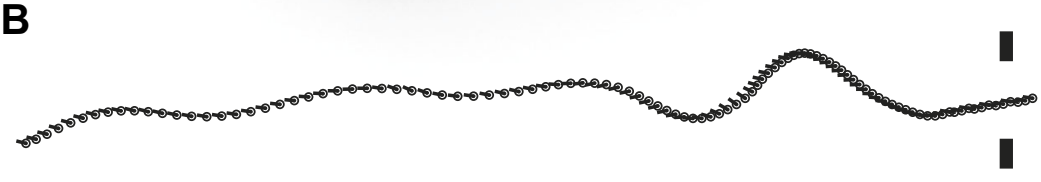

$d=550 \mathrm{~mm}$
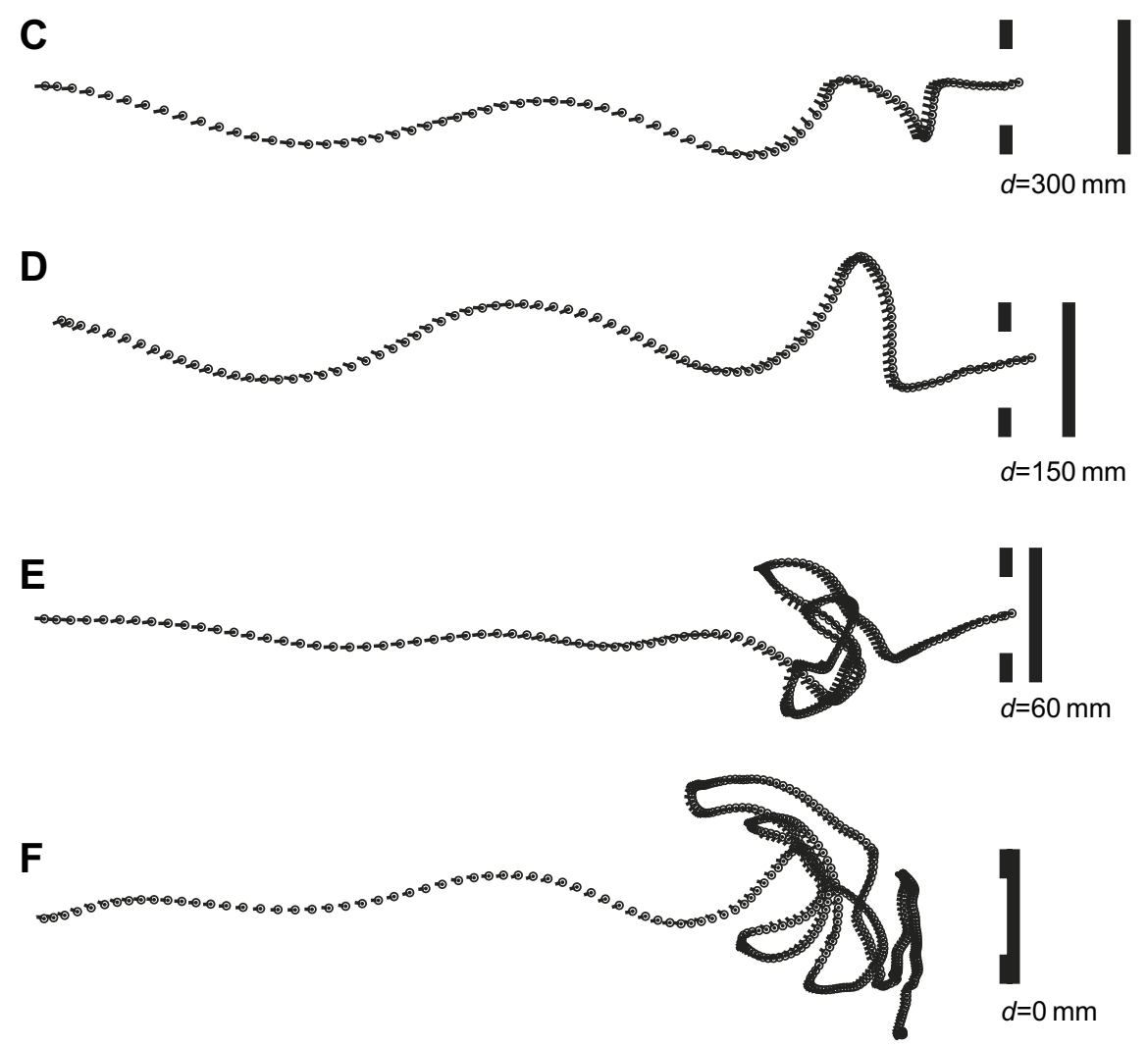

direct flights along the tunnel. Only flights of individuals that appeared to be returning from foraging trips, considered as those bees that made a steady and direct flight towards the gap, were used for analysis. At least one such flight was observed every minute. Among all the flights recorded, the body length of the individual bee varied by less than $5 \%$, indicative of the nominally constant altitude maintained during the entire flight. In order to attenuate digitizing error, the flight trajectories were passed through a $30 \mathrm{~Hz}$ secondorder Butterworth filter. Flight speed along the longitudinal and lateral directions was estimated by differentiating the flight trajectory along the respective axis and applying a coordinate transformation matrix to obtain body-centered values. Heading orientation was calculated with respect to the flight tunnel using the right-hand rule. As the flights were recorded from a single perspective, pitch and roll could not be measured.
Geometric optic flow, measured as the angular displacement of the vector between an arbitrary point in space and the retina due to relative motion (Eqn 1), was calculated in MATLAB using the flight trajectory and flight tunnel geometry. Here, for each flight in all conditions, the true optic flow was calculated using the respective flight trajectory, assuming constant head-body alignment, a spherical eye and the retina approximated as a point. As flight videos were recorded using one camera, the bees were assumed to fly at mid-height of the tunnel. This was a reasonable assumption and it permitted the estimation of optic flow along the elevation and azimuthal axes. A similar approach has been implemented in numerous previous studies (Bertrand et al., 2015; Serres and Ruffier, 2017; van Breugel et al., 2014).

The true geometric optic flow was calculated by first discretizing the ommatidium as a ray emanating from the point retina. The 
compound eye was considered to consist of 10,000 rays equally spaced along the elevation and azimuth of the spherical eye. For each instant (a frame from the high-speed video), the intersection point between the ray from the bumblebee's retina and a solid feature in the environment, such as a check on the flight-tunnel walls, was identified $\left(\vec{u}_{\theta, \varphi, t}\right)$. Subsequently, the properties of the ray connecting this intersection point in the environment and the bumblebee's retina at a new spatial location and orientation based on a body-fixed coordinate system in the next time instant was calculated $\left(\vec{v}_{\theta, \varphi, \Delta t}\right)$. The total geometric optic flow for this ommatidium was therefore the angle between the two rays separated by the time between the two frames - in this case $1 / 200$ frames s $^{-1}$ (see Eqn 1). This process was repeated for all rays of the compound eye and once again repeated by considering each frame with its subsequent frame to evaluate the geometric optic flow along the trajectory. Yaw rotations of the bee were accounted for by adding the yaw rate to the geometric optic flow based on the body coordinate system. While our optic flow estimation is simplified and differs in some respects from the mechanism of optic flow estimation in bumblebees, the underlying principle remains similar and thus the overarching conclusions derived from the analysis here can be considered valid:

$$
\dot{\alpha}_{(\theta, \varphi, t)}=\cos ^{-1}\left(\frac{\vec{u}_{(\theta, \varphi, t)} \cdot \vec{v}_{(\theta, \varphi, t+\Delta t)}}{\left(\left\|\vec{u}_{(\theta, \varphi, t)}\right\| \cdot\left\|\vec{v}_{(\theta, \varphi, t+\Delta t)}\right\|\right)}\right) \cdot \mathrm{FR},
$$

where $\dot{\alpha}_{\theta, \varphi, t}$ is the geometric optic flow, $\theta$ and $\varphi$ are the elevation and azimuth of the ray from the retina at time instant $t, \vec{u}_{\theta, \varphi, t}$ is the vector from the retina to the intersecting solid point in the environment and $\vec{v}_{\theta, \varphi, \Delta t}$ is the vector connecting that intersection point in the environment and the retina an incremental time later. The frame rate of the camera (FR, in frames $\mathrm{s}^{-1}$ ) in this case was $200 \mathrm{~Hz}$.

A total of 100 flights ( 20 per condition $\times 5$ conditions) were recorded and analyzed in this study. Data normality was tested using the Lilliefors test in MATLAB. The null hypothesis (data are normally distributed) was validated for all datasets that were statistically compared. Statistical significance of the variation in quantities between experimental conditions was tested using a one-way ANOVA; a Tukey post hoc test confirmed significant conditions within the group. For comparison of quantities within each experimental condition, a paired $t$-test was used to assess statistical significance. $P<0.05$ was considered to indicate a statistically significant difference between the quantities being tested, and for cases where multiple comparisons were made within each experimental condition, the Bonferroni correction was implemented. No statistical analysis was conducted for the condition where a glossy white background was placed immediately in the rear of the gap and only five flights were permitted; in this case, the analysis was qualitative.

\section{RESULTS}

Upon entering the flight tunnel, all bees took off and flew smoothly as they approached the unfamiliar wall blocking their flight path. For all experiment conditions en route to the gap, the flight trajectories of the bees were not straight, but contained some smooth lateral movements (see Fig. 1B-F). The bees performed increased lateral maneuvers closer to the gap as the distance between the gap and rear wall was reduced (see Fig. 1D-F). For all conditions, when the distance between the gap and the rear wall was $<60 \mathrm{~mm}$, the bees engaged in forward-facing crescent-shaped maneuvers close to the gap $(<100 \mathrm{~mm}$; see Fig. 1E), prior to passing through it. When the gap was not present, i.e. the rear wall was directly adjacent to the gap, the bees continued to perform crescent-shaped flights while facing forward close to the center of the tunnel, with increasing arc size. None of the bees attempted to pass through in this condition (see Fig. 1F). This also included the condition when a non-textured white wall was placed immediately adjacent to the gap, similar to the $d=0 \mathrm{~mm}$ condition (data not shown). An apparent increase in the sideward component of the flight path was evident with decreasing distance between the gap and rear wall. These crescent-shaped flight paths bear nominal similarity to learning flights of bumblebees after they leave their nest hole and are assumed to be gathering information about their surroundings (e.g. Lobecke et al., 2018; Philippides et al., 2013).

In order to quantify the flight trajectories, the tunnel was binned into six segments and the longitudinal flight speed of the bees within each bin was calculated (see Fig. 2A). Irrespective of the distance between the gap and rear wall, the flight speed among the different individuals remained statistically similar when they were up to $375-525 \mathrm{~mm}$ to the gap (Fig. $2 \mathrm{~A} ; F_{4,95}=0.79, P=0.55$ ). The flight speed of the bees within this region was nominally similar to those reported by Baird et al. (2010), where a similar sized tunnel was used. However, there was considerable variation in flight speed (up to $1.5 \times$ variation in magnitude) among the different flights across all conditions. At distances closer than $375 \mathrm{~mm}$ to the gap, the bees approached the gap while steadily decelerating wherein the rate of deceleration was dependent on the distance between the gap and background (Fig. 2A). Comparing the longitudinal flight speed of the bees along different sections of the flight tunnel, for all conditions when the distance between the gap and rear wall was $<150 \mathrm{~mm}$, a significant reduction in the mean longitudinal speed of the bees occurred when they were in the distance range 375$225 \mathrm{~mm}$ compared with when they were $375-525 \mathrm{~mm}$ to the gap $(d=150 \mathrm{~mm} P=0.043, d=60 \mathrm{~mm} P=0.029$ and $d=0 \mathrm{~mm} P=0.0068$; see Fig. 2A). With $d>150 \mathrm{~mm}$, the longitudinal velocity of the bees in the distance range $375-225 \mathrm{~mm}$ to the gap was lower compared with when they were between 375 and $525 \mathrm{~mm}$ to the gap; however, the reduction was not statistically significant $(d=300 \mathrm{~mm} P=0.068$ and $d=550 \mathrm{~mm} P=0.075$ ). For $d=550,300$ and $150 \mathrm{~mm}$, the flight speed of the bees was significantly reduced only when they were 225-150 mm to the gap compared with when they were 375$225 \mathrm{~mm}$ to the gap $(d=550 \mathrm{~mm} P=0.022, d=300 \mathrm{~mm} P=0.046$ and $d=150 \mathrm{~mm} P=0.013$ with Bonferroni correction; Fig. 2A). When the bees were $150-75 \mathrm{~mm}$ to the gap, there was a monotonic reduction in their mean speed with decreasing distance between the gap and rear wall $\left(F_{4,95}=3.18, P=0.02\right)$.

For all experimental conditions, the mean absolute lateral speed of the bees was small but non-zero at large distances to the gap $(>150 \mathrm{~mm})$ and monotonically increased as they approached the gap ( $F_{4,95}=5.66, P=0.003$; see Fig. $2 \mathrm{~B}$ ). Unlike the longitudinal speed, the mean absolute lateral flight speed did not become significantly different across the different conditions until the bees were in the distance range $225-150 \mathrm{~mm}$ to the gap; within this region of the tunnel, the bees' lateral velocity was largest when the rear wall was adjacent to the gap (comparing $d=500$ and $0 \mathrm{~mm}, P=0.00935$; Fig. 2B). Similar to the forward speed, the rate of increase in the mean lateral speed of the bees was also dependent on the distance between the gap and rear wall. The ratio of mean lateral speed to the total speed of the bees summarily increased the closer the bees got to the gap (Fig. 2C). For the non-passable condition with $d=0$, the bees mostly moved laterally at distances $75-0 \mathrm{~mm}$ to the gap over the duration of the recording (Fig. 2B,C). The lateral extent of the tunnel that the bees covered within each segment also increased as they got closer to the gap (Fig. 1B-F).

To unravel the mechanics of the lateral movements and the flight maneuvers performed close to the gap for conditions where the 


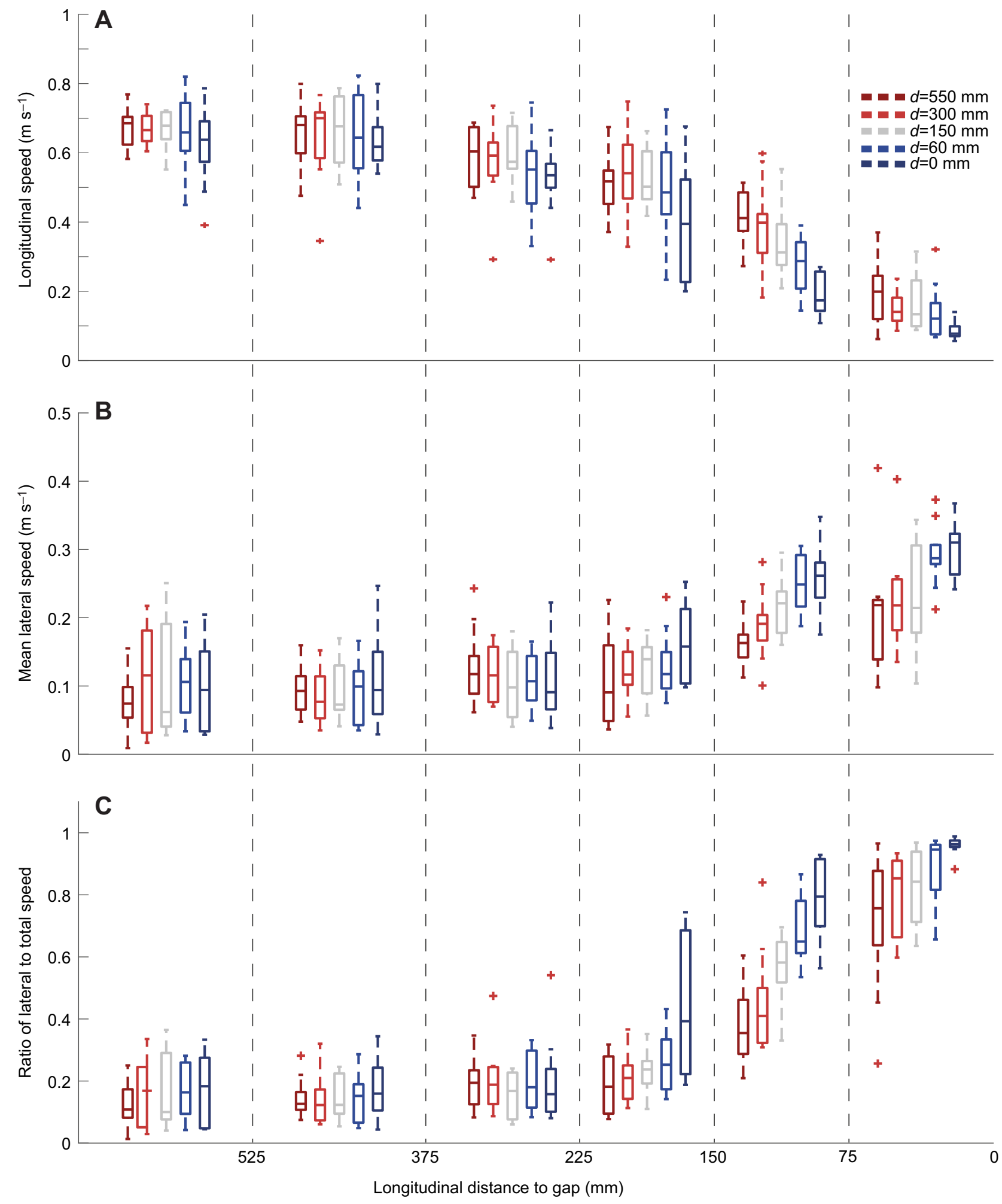

Fig. 2. Flight speed analysis. The flight tunnel was binned into six sections leading to the gap. (A) The absolute mean longitudinal speed of the bees

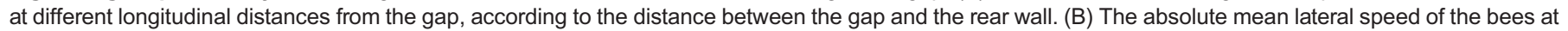
different sections of the flight tunnel. (C) Ratio of the mean absolute lateral speed and the total speed.

distance between the gap and rear wall was small, the total acceleration of the bees in the body coordinate system at three different segments along the tunnel was represented as a rose histogram (Fig. 3). The length of each angular column in the rose histogram indicates the probability of the total acceleration of the bee being within the range of the respective bin. This was done for the $d=0 \mathrm{~mm}$ condition where lateral movements were most significant. The angle between the total acceleration and the long axis of the body was binned into 20 segments of 18 deg width for all recorded flights. When the bees were 300-200 $\mathrm{mm}$ from the gap, 

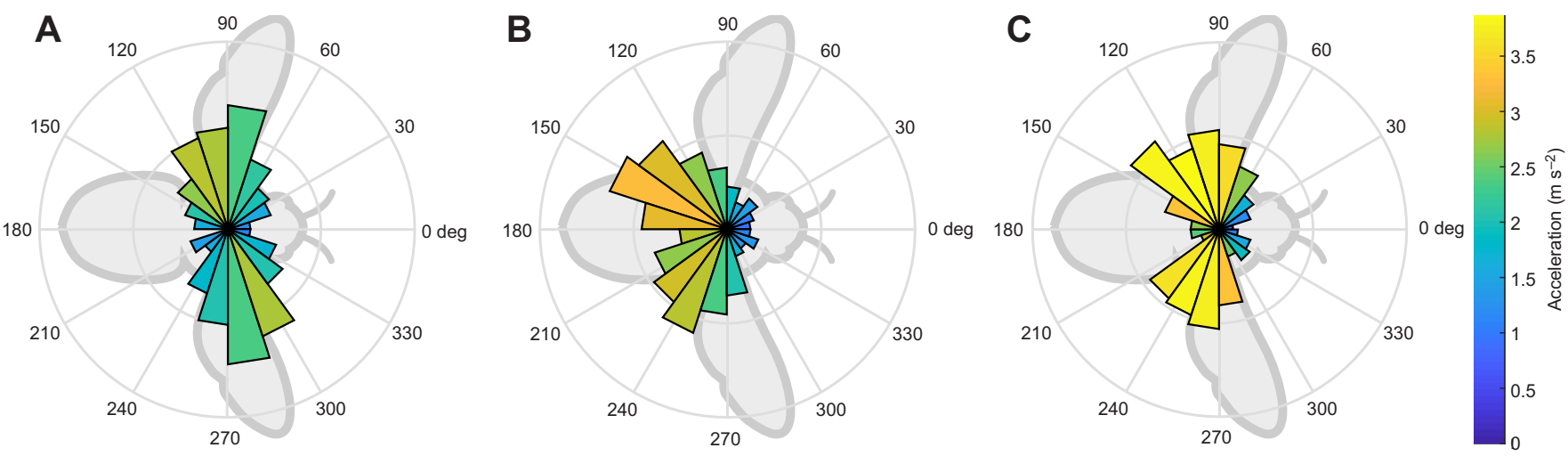

Fig. 3. Rose histogram of the total acceleration with respect to the long body axis among all flights at different sections of the flight tunnel. (A) $300-$ $200 \mathrm{~mm}$, (B) 200-100 mm and (C) 100-0 mm from the gap. The length of each angular column in the rose histogram indicates the probability of the total acceleration of the bee being within the range of the respective bin. The hue represents the magnitude of mean acceleration within the respective angular bins.

the total acceleration was oriented laterally with respect to their body and small in longitudinal direction. Between 200 and $100 \mathrm{~mm}$ to the gap, the bees began decelerating (Fig. 3B), and this is evident in the acceleration histogram where the total acceleration was distributed mostly laterally with a rearward skew (second and third quadrant). In the proximal regions of the gap (100-0 mm), the total acceleration was predominantly orientated orthogonal to the body long axis (Fig. 3C). The magnitude of acceleration, as indicated by the hue in Fig. 3, was also lowest when the bees were far from the gap. It progressively increased near the gap, where the flight was characterized by the largest accelerations oriented nominally orthogonal to the longitudinal axis (Fig. 3).

\section{Optic flow analysis}

The above results clearly revealed that the flight behavior of bumblebees is strongly affected by the distance between the gap and the rear wall. A likely cue providing information about the spatial layout under the different conditions is the optic flow within the gap and in the adjacent parts of the visual field. Therefore, we determined the optic flow difference between the inside and the outside of the gap. The mean of the absolute difference of total geometric optic flow across the inside and outside edge of the gap ( $\pm 12 \mathrm{~mm}$ along the gap edge) is presented in Fig. 4A. As expected, the difference in optic flow across the edge of the gap was low when the bees were far from the gap and it progressively increased as the bees neared the gap. When the bees were $225-150 \mathrm{~mm}$ to the gap, the difference in mean optic flow across the edge increased significantly with decreasing distance between the gap and the rear wall $\left(F_{4,95}=0.79, P<10^{-3}\right)$. However, in the near vicinity of the gap (75-0 $\mathrm{mm})$, the mean optic flow difference across the edge of the gap was not statistically significant across the $d=550-150 \mathrm{~mm}$ conditions $\left(F_{4,95}=0.28, P=0.74 ;\right.$ Fig. $\left.4 \mathrm{~A}\right)$. The optic flow difference for $d=0$ was non-zero (Fig. 4A) because of the offset in the wall position due to their thickness $(1.5 \mathrm{~mm})$. The mean optic flow on the wall containing the gap and the rear wall when the bees were $<150 \mathrm{~mm}$ to the gap for all conditions is presented in Fig. 4B-F. A sharp optic flow discontinuity at the edge of the gap is present when the rear wall is further away from the gap. Consequently, the distinctness of the gap in the optic flow profile clearly decreases with decreasing distance between the gap and the rear wall in the different experiment conditions.

The difference in optic flow across the edge of the wall was normalized with respect to the optic flow $12 \mathrm{~mm}$ outside the gap edge, on the wall containing the gap, to reveal the mean motion contrast for the different conditions (see Fig. 5A). For all flights, only the flight trajectories of the bees when they were $75-0 \mathrm{~mm}$ to the gap were considered for the contrast estimation. A high motion contrast was present only when the distance between the gap and rear wall was large and it monotonically decreased with decreasing distance. Concomitant with the decreasing motion contrast, an opposite trend was noted in the time spent by the bees in the near vicinity of the gap (75-0 mm) before passage (Fig. 5B). Bees spent a longer time $75-0 \mathrm{~mm}$ to the gap as the distance between the gap and rear wall decreased from 550 to $0 \mathrm{~mm}$ (Fig. 5B). For the extreme condition when gap passage was impossible $(d=0)$, the time spent was not calculated as the bees continued to traverse laterally and no attempts to pass were made. We can conclude that the bees spend more time exploring the situation close to the gap when the optic flow contrast across the gap and, thus, the distance between the gap and the rear wall gets smaller. At the same time, they increase the lateral velocity as a means to increase absolute difference in optic flow across the edge of the gap.

\section{DISCUSSION}

Despite their tiny brains, bumblebees and other eusocial insects display a remarkable capacity for navigation through inherently complex environments. The most elemental aspect for locomotion through a cluttered terrain is the identification of a gap between obstacles and subsequently assessing passability. In our experiments, we sought insight into the salient mechanisms utilized by bumblebees to identify a gap when presented with an unexpectedly altered environmental situation relative to the conditions of an unobstructed tunnel that the bees were familiar with. Thus, the bumblebees could not learn the gap properties. By considering only bees that were used to returning to the hive through a familiarized unobstructed flight tunnel, we exploited the high motivational state of the animal in identifying a route through the altered environment that required passage through the gap. In an alternative setup when the gap was presented to bees that were en route from the hive to the foraging chamber, the bees were much less amenable to the experimental paradigm and chose to return to the safety of the hive.

\section{Gap approach}

When the bees were far from the gap, their flight trajectory seemed to be driven by the well-established mechanism of equalizing bilateral optic flow. As the spatial information on the sidewalls of the tunnel was similar - a random checkerboard pattern and a 

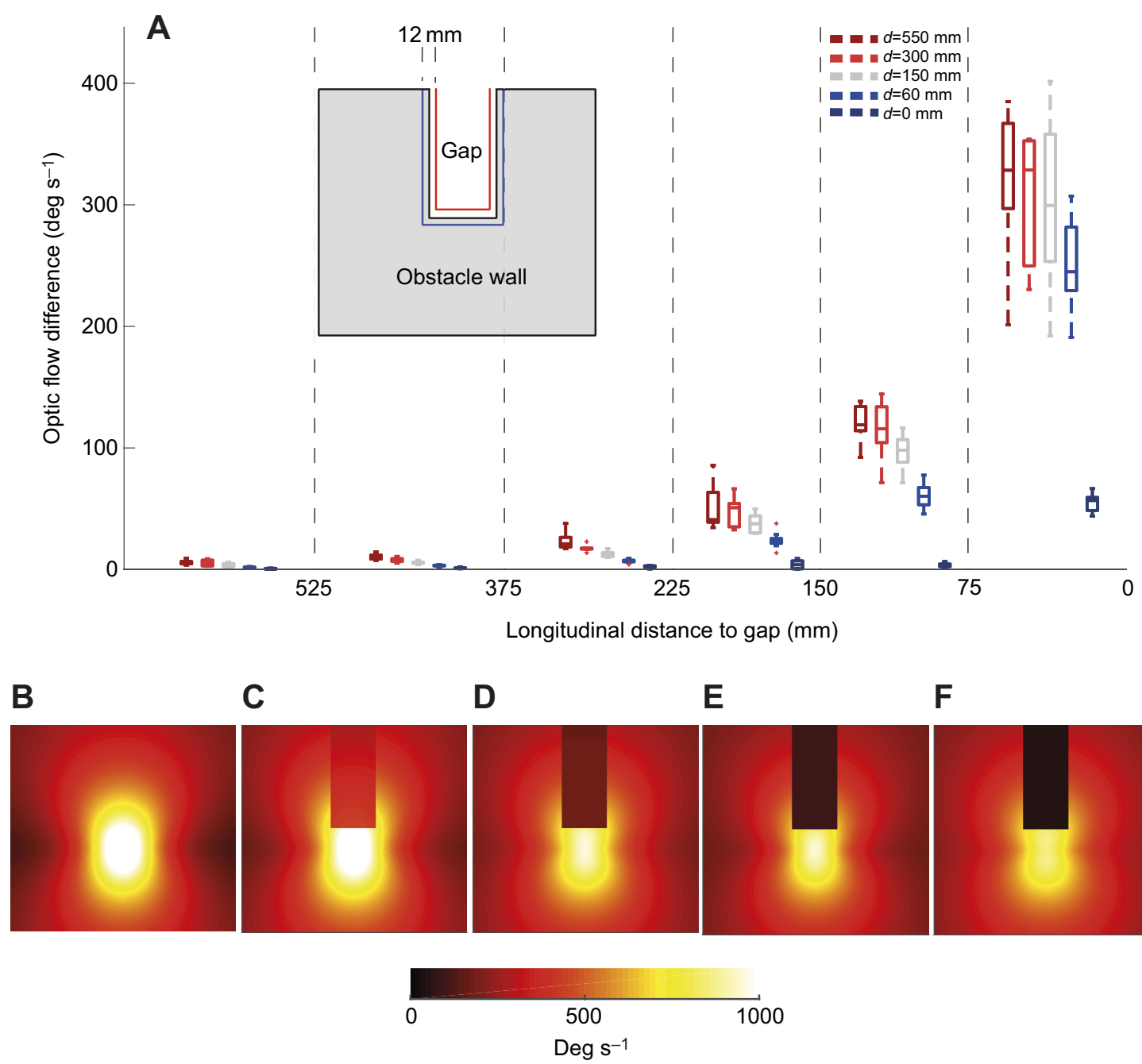

Fig. 4. Optic flow within the gap. (A) The mean of the absolute difference in total optic flow across the inside (red line in inset) and outside edge of the gap (blue line in inset), which are separated by $12 \mathrm{~mm}$ along the gap edge, at different sections of the flight tunnel. (B-F) Heat map showing the mean geometric optic flow over the wall containing the gap when $d=0 \mathrm{~mm}(B), 60 \mathrm{~mm}(C), 150 \mathrm{~mm}(\mathrm{D}), 300 \mathrm{~mm}(\mathrm{E})$ and $550 \mathrm{~mm}(\mathrm{~F})$ across all flight trajectories when the bees were $<75 \mathrm{~mm}$ from the gap. The geometric optic flow was calculated taking into account both the longitudinal and lateral translation as well as yaw rotations of the bees as they approached the gap.

nominally homogeneous illumination - the bees flew close to the centerline of the flight tunnel. This is a familiar feature observed in a number of previous studies that have utilized flight tunnels to study insect and bird flight (Bhagavatula et al., 2011; Schiffner et al., 2014; Srinivasan, 2010). Under the conditions of our experiments, the bees flew at around $0.7 \mathrm{~m} \mathrm{~s}^{-1}$ in the far field of the gap, which was similar to speeds measured by Baird et al. (2010) where a similar experimental paradigm was used. Smooth sideward motion interlaced the longitudinal velocity in the bees (Fig. 2B-F); this lateral 'casting' motion is also a common feature noted in previous experiments on bumblebee flight (Chang et al., 2016; Dyhr and Higgins, 2010; Linander et al., 2015; Ravi et al., 2013).

At around $375 \mathrm{~mm}$ from the gap, evidence of changes in behavior were first noted as a reduction in flight speed (Fig. 2A). In this region of the flight tunnel, the angle subtended by the obstructing wall containing the gap and the gap itself was 36-42 deg and 9$14 \mathrm{deg}$, respectively. Baird et al. (2010) reported that bumblebees modulate their flight speed using the frontal optic flow and showed that bees responded to abrupt changes in flight tunnel width when it subtended between 23 and 30 deg on the retina, which is consistent with our data. The bees could be responding to the obstructing wall, the properties of the gap or a combination of the two. In eliciting a change in flight speed, there appeared to be a combined influence of the obstructing wall and the distance between the gap and rear wall when the bees were between 375 and $225 \mathrm{~mm}$ from the gap. In this region, the first consistent reduction in flight speed across all conditions compared with when they were $>375 \mathrm{~mm}$ from the gap was noted; however, it was statistically significant only when $d<60 \mathrm{~mm}$ (see Fig. 2A). Within this region, the mean optic flow difference across the edge of the gap was $60-120 \mathrm{deg} \mathrm{s}^{-1}$ when $d=550 \mathrm{~mm}$, while the optic flow on the wall along the gap edge was only 3-12 deg s${ }^{-1}$ for the extreme non-passable condition $(d=0)$. The deceleration of the bees for the non-passable condition may be considered as that elicited purely by the obstructing wall. This suggests that in our experimental paradigm the prominence of the gap modulated the approach flight speed of the bees. Comparatively, when they were $<225 \mathrm{~mm}$ from the gap, further reduction in flight speed appeared to be mainly influenced by the distance between the gap and rear wall (see Fig. 2A). 

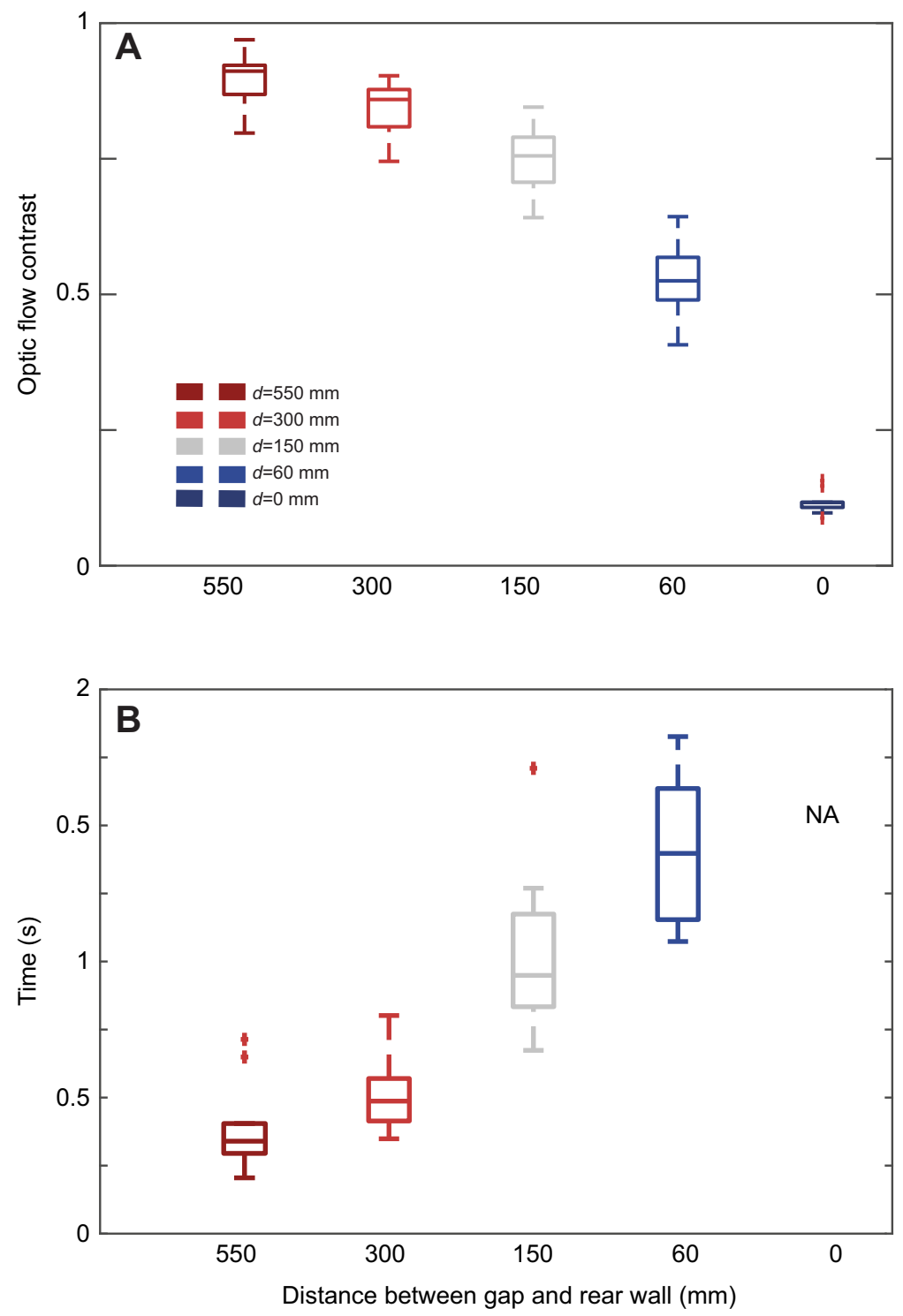

Fig. 5. Motion contrast and time in the vicinity of the gap. (A) Optic flow contrast was measured as the ratio between the mean difference in optic flow across the edge of the gap (the mean difference in optic flow along the red and blue lines in the inset in Fig. 4A) and the mean optic flow along the outer edge of the gap (mean optic flow along the blue line). (B) Time spent by the bees in the vicinity of the gap $(<100 \mathrm{~mm})$ for the different experimental conditions.

\section{Gap perception}

In order to cross the gap, the bumblebees have to detect it and assess its passability; namely, the distance between the gap and the rear wall. As the distance between the gap and the rear wall decreases, the texture surrounding the gap and that of the rear wall, as seen by the bee, become more similar, and the optic flow at the edge of the gap decreases. Assessing the passability becomes, therefore, more difficult when the distance between the gap and the rear wall decreases. Thus, the bumblebee may need more time either to make its decision or to actively shape its flight style by generating sidewise movements to enhance the differences in the optic flow between the surroundings of the gap and the rear wall. We will discuss the behavior of the bumblebee in front of the gap from the perspective of both hypotheses: (1) that bees move in a manner that enhances the dissimilarities between the surroundings of the gap and the rear wall and (2) that they rely on texture differences.

For all conditions, concomitant with decelerating longitudinal flight speed the bees increased their lateral speed as they neared the gap (Fig. 2). They also increased lateral speed significantly as the distance between the gap and rear wall was decreased (Figs 2 and 3). The total accelerations during these lateral maneuvers were higher closer to the gap and mainly oriented normal to the body long axis (Fig. 3). In such cases, the bees were 'side slipping', performed by rolling their body to redirect their aerodynamic force vector in the direction of movement, similar to a helicopter (Ravi et al., 2016; Taylor, 2001). Such body roll-mediated lateral maneuvers are usually coupled with synchronous counter-rotations of the head to maintain a stable visual field (Boeddeker and Hemmi, 2010). Performing lateral maneuvers with only a little body yaw significantly increases the lateral translational optic flow.

Flies, wasps and a number of other volant insects actively shape the optic flow on the retina by modulating their head and body trajectory to increase the translatory component while minimizing rotations (Egelhaaf et al., 2014). Optic flow derived from translation contains information on the relative distance between environmental features such as obstacles while optic flow from rotations lacks this vital information, which is why volant insects tend to restrict rotations to rapid saccades (Egelhaaf et al., 2012). Bumblebees flew in our tunnel with minimal yaw rotations (Fig. 1B-F), thus increasing translational optic flow. Optic flow derived through pure longitudinal motion is not very sensitive to distance differences in the frontal visual field, as the flow vectors are small close to the focus of expansion. Increased optic flow sensitivity to distance differences between environmental features in the frontal visual field can be achieved, however, through 
lateral translation (motion parallax) (Collett, 2002; Srinivasan et al., 1990; Sobel, 1990; Wallace, 1959). Even when the bees were seemingly uninfluenced by the obstacles ( $>375 \mathrm{~mm}$, Fig. 2$)$, their flight path consisted of smooth lateral movements, i.e. casting. The significance of these voluntary lateral movements performed by the bees (Ravi et al., 2016) is unclear. However, they are likely to be used to increase lateral translational optic flow and, thus, to aid depth perception.

A consequence of the increased lateral translations performed by the bees in the proximity of the gap is the large difference in optic flow across the edges of the gap, thereby increasing their salience (Fig. 4B-F). An example of active shaping of optic flow through flight maneuvers can be seen by comparing, for the $150 \mathrm{~mm}$ rear wall condition, the generated optic flow difference across the gap edge with the other conditions (Fig. 4A). For this condition and when the distance of the bees to the gap was $>75 \mathrm{~mm}$, the difference in optic flow across the gap edge was significantly lower than for the $>150 \mathrm{~mm}$ rear wall conditions. However, when the bees were close to the gap $(<75 \mathrm{~mm})$, the optic flow difference across the gap edge was $200-400 \mathrm{deg} \mathrm{s}^{-1}$, similar to the $550 \mathrm{~mm}$ rear wall condition as a consequence of the bees' increased lateral maneuvers (Fig. 4A). A similar trend was observed for the $60 \mathrm{~mm}$ rear wall condition; however, the optic flow difference then remained significantly lower even when the bees were near the gap $(<75 \mathrm{~mm})$. Active maneuvering in order to discern depth and increase salience of the edges appears to be a compensatory strategy of the bees to the changing distance of the rear wall. Bumblebees, wasps, honeybees and other insects have been observed to perform nominally similar flight maneuvers, which consist of large lateral components, for instance, during their learning flights after leaving an attractive goal location, such as a food source or a nest hole (Dittmar et al., 2010; Kern et al., 1997; Lobecke et al., 2018; Zeil, 1996). Here, we showed that bees actively modulate such behavior in a gap perception context and it appears to depend on the salience of the gap.

In this case, bees are likely utilizing a combination of information about the velocity of self-motion, which is related to the input motor signals, and the relative optic flow in discerning the gap salience. The monotonic increase in lateral velocity with decreasing distance between the gap and rear wall does not increase at the same rate until the extreme condition when the gap and rear wall are adjacent (Fig. 2C). Our results suggest that it is likely that there exists a threshold dependent on the salience of the gap. We believe this threshold might reflect passability based upon identifying gap properties including depth through lateral maneuvering. If the bees cannot assess safe passage, they might resort to searching for alternative gaps in the environment or may fly back. The nominally crescent-shaped flight pattern of the bees close to the gap for the cases where the rear wall was $<60 \mathrm{~mm}$ bears similarity to searching flights and orientation or learning flights performed by bumblebees upon their first departure from their nest hole, where they are assumed to probe the layout of the behaviorally relevant nest hole environment (Lobecke et al., 2018; Philippides et al., 2013; Riabinina et al., 2014).

In contrast to enhancing optic flow differences by an active behavioral vision strategy, the observed sideways movements of the bees may be a byproduct of a longer decision time (or hesitation) required to assess the passability of the gap as the distance between the gap and the rear wall decreases, even if the bees do not gather additional information during these movements. However, if the bees, because of their limited hovering abilities, would just have to generate sideways motion to have more time for processing the already gathered information, there is no reason to increase the ratio between lateral and forward motion when the distance between the gap and rear wall decreases. As this ratio has been observed to increase, the bees do not only need more time to make their decision but also actively change their behavior in a way that enhances the difference in optic flow between the gap and the rear wall. Because texture dissimilarities do not become more evident with increased lateral motion, we regard the active vision hypothesis to be most plausible, although further experiments need to be carried out (e.g. by actively moving the rear wall while the bee is approaching the gap) to further substantiate this hypothesis.

A number of other factors are also likely to influence the flight pattern of the bees in this condition, including the geometry of the flight tunnel and the obstacles. However, the similarity between the flights when negotiating the gap in our flight tunnel to learning and searching flights of bees observed in the context of local homing behavior merits further investigation. We suggest that in both situations these characteristic meandering lateral flight maneuvers serve the same basic purpose, i.e. probing the spatial layout of the environment.

The few conditions in our experimental analysis where a white background was placed immediately to the rear of the gap created a scenario where a high optic flow difference across the gap was present but there was nearly zero optic flow within the gap. In this condition, none of the bees attempted to pass, suggesting that apart from the difference in optic flow across the edges of the gap, a nonzero optic flow within the gap may be one of the conditions necessary for passage, because optic flow inside the gap will provide information about the spatial situation behind the gap. Additionally, the homogeneous lighting used in our setup might not have created the necessary brightness difference across the edges of the gap to elicit a brightness-based response. Further investigations are necessary to identify the presence of such virtuosic strategies.

\section{Time to decision}

When flying within a complex cluttered environment, an animal constantly needs to evaluate the environmental features confronting it and make decisions that influence the flight course. Bees spend significant time in the near vicinity of the gap while performing the rapid lateral maneuvers (Fig. 5B). The consistent repeated flights of the bees, especially when the rear wall was $<60 \mathrm{~mm}$ from the gap, suggest that through these flights the bees not only discern the gap geometry but also evaluate passability. Once the potential for safe passage is established, traversal through the gap occurs. Measuring the time spent by the bees within the region where most of the lateral maneuvers occur might provide an indication of the time taken by the bees to arrive at a decision. Among all flights recorded, none of the bees performed abrupt corrective maneuvers once gap traversal had commenced, indicating that decision making occurs ahead of the gap.

The optic flow contrast appears to be a critical parameter because of a strong and direct inverse relationship with respect to the time spent by the bees evaluating the gap (Fig. 5). Through the repeated lateral movements, the bees appear to establish the salience of the gap and confidence about the geometry by actively generating visual information about passability. The smaller the perceived gap, the larger the sideways velocities in order to increase optic flow contrast and the longer the bees probe the environment to potentially increase their confidence about the situation. As a consequence, decision making is delayed. Other factors such as familiarity and experience, though unlikely to play a significant role in these experiments, are also likely to influence the bees' decision time in assessing gap properties and passability in their natural environment (Zhang and Srinivasan, 1994), and further experiments are necessary to quantify the influence of these factors on the neural and biophysical mechanics of locomotion through spatially complex environments. 


\section{Acknowledgements}

We thank the anonymous reviewers whose comments significantly improved the quality of the manuscript.

\section{Competing interests}

The authors declare no competing or financial interests.

\section{Author contributions}

Conceptualization: S.R., T.S., L.M., A.F., M.E.; Methodology: S.R., O.B., T.S., L.M., C.D., A.F., M.E.; Software: S.R.; Validation: S.R.; Formal analysis: S.R., A.F. Investigation: S.R., O.B., T.S., L.M., C.D.; Resources: S.R., O.B.; Writing - original draft: S.R., O.B., C.D., A.F., M.E.; Writing - review \& editing: S.R., O.B., T.S., L.M., C.D., A.F., M.E.; Supervision: M.E.; Project administration: S.R., M.E.; Funding acquisition: M.E.

\section{Funding}

This project was funded by the Alexander von Humboldt Stiftung through a Research Fellowship awarded to S.R. as well as by the Deutsche Forschungsgemeinschaft (DFG grant number OJNB: EG82/19-1) and by the Cluster of Excellence EX 277 Cognitive Interaction Technology (CITEC), funded by Deutsche Forschungsgemeinschaft.

\section{References}

Baird, E. and Dacke, M. (2012). Visual flight control in naturalistic and artificia environments. J. Comp. Physiol. A. Neuroethol. Sens. Neural. Behav. Physiol. 198, 869-876.

Baird, E. and Dacke, M. (2016). Finding the gap: a brightness-based strategy for guidance in cluttered environments. Proc. Biol. Sci. 283, 20152988.

Baird, E., Kornfeldt, T. and Dacke, M. (2010). Minimum viewing angle for visually guided ground speed control in bumblebees. J. Exp. Biol. 213, 1625-1632.

Baird, E., Boeddeker, N., Ibbotson, M. R. and Srinivasan, M. V. (2013). A universal strategy for visually guided landing. Proc. Natl. Acad. Sci. USA 110 18686-18691.

Bertrand, O. J. N., Lindemann, J. P. and Egelhaaf, M. (2015). A bio-inspired collision avoidance model based on spatial information derived from motion detectors leads to common routes. PLOS Comput. Biol. 11, e1004339.

Bhagavatula, P. S., Claudianos, C., Ibbotson, M. R. and Srinivasan, M. V. (2011) Optic flow cues guide flight in birds. Curr. Biol. 21, 1794-1799.

Boeddeker, N. and Hemmi, J. M. (2010). Visual gaze control during peering flight manoeuvres in honeybees. Proceedings. Biol. Sci. 277, 1209-1217.

Braun, E., Geurten, B. and Egelhaaf, M. (2010). Identifying prototypica components in behaviour using clustering algorithms. PLoS ONE 5, e9361.

Braun, E., Dittmar, L., Boeddeker, N. and Egelhaaf, M. (2012). Prototypica components of honeybee homing flight behavior depend on the visua appearance of objects surrounding the goal. Front. Behav. Neurosci. 6, 1.

Chang, J. J., Crall, J. D. and Combes, S. A. (2016). Wind alters landing dynamics in bumblebees. J. Exp. Biol. 219, 2819-2822.

Collett, T. S. (2002). Insect vision: controlling actions through optic flow. Curr. Biol. 12, R615-R617.

Crall, J. D., Ravi, S., Montcastle, A. A. Combes, S. A. (2014). Bigger but not always better: Tradeoffs between maneuverability and flight speed with body size in bumblebees. Annual Meeting of the Society for Integrative and Comparative Biology 54, E44-E44.

Crall, J. D., Ravi, S., Mountcastle, A. M. and Combes, S. A. (2015). Bumblebee flight performance in cluttered environments: effects of obstacle orientation, body size and acceleration. J. Exp. Biol. 218, 2728-2737.

Dittmar, L., Stürzl, W., Baird, E., Boeddeker, N. and Egelhaaf, M. (2010). Goa seeking in honeybees: matching of optic flow snapshots? J. Exp. Biol. 213 2913-2923.

Dudley, R. (2002). The Biomechanics of Insect Flight: Form, Function, Evolution Princeton University Press.

Dyhr, J. P. and Higgins, C. M. (2010). The spatial frequency tuning of optic-flowdependent behaviors in the bumblebee Bombus impatiens. J. Exp. Biol. 213 1643-1650.

Egelhaaf, M., Kern, R., Lindemann, J. P., Braun, E. and Geurten, B. (2010) Active vision in blowflies: strategies and mechanisms of spatial orientation. In Flying Insects and Robots (D. Floreano, J.-C. Zufferey, M. V. Srinivasan and C. Ellington), pp. 51-61. Springer

Egelhaaf, M., Boeddeker, N., Kern, R., Kurtz, R. and Lindemann, J. P. (2012). Spatial vision in insects is facilitated by shaping the dynamics of visual input through behavioral action. Front. Neural Circuits 6, 108.
Egelhaaf, M., Kern, R. and Lindemann, J. P. (2014). Motion as a source of environmental information: a fresh view on biological motion computation by insect brains. Front. Neural Circuits 8, 127.

Kern, R., Egelhaaf, M. and Srinivasan, M. V. (1997). Edge detection by landing honeybees: behavioural analysis and model simulations of the underlying mechanism. Vision Res. 37, 2103-2117.

Kern, R., Boeddeker, N., Dittmar, L. and Egelhaaf, M. (2012). Blowfly flight characteristics are shaped by environmental features and controlled by optic flow information. J. Exp. Biol. 215, 2501-2514.

Knaden, M. and Graham, P. (2016). The sensory ecology of ant navigation: from natural environments to neural mechanisms. Annu. Rev. Entomol. 61, 63-76.

Linander, N., Dacke, M. and Baird, E. (2015). Bumblebees measure optic flow for position and speed control flexibly within the frontal visual field. J. Exp. Biol. 218 1051-1059.

Lobecke, A., Kern, R. and Egelhaaf, M. (2018). Taking a goal-centred dynamic snapshot as a possibility for local homing in initially naïve bumblebees. J. Exp. Biol. 221, jeb.168674.

Mertes, M., Dittmar, L. and Egelhaaf, M. (2015). Bumblebee homing: the fine structure of head turning movements. PLoS Biol.

Mirwan, H. B. and Kevan, P. G. (2013). Social learning in bumblebees (Bombus impatiens): worker bumblebees learn to manipulate and forage at artificial flowers by observation and communication within the colony. Psyche A J. Entomol. 2013, 1-8.

Monteagudo, J., Lindemann, J. P. and Egelhaaf, M. (2017). Head orientation of walking blowflies is controlled by visual and mechanical cues. J. Exp. Biol. 220 4578-4582.

Ong, M., Bulmer, M., Groening, J. and Srinivasan, M. V. (2017). Obstacle traversal and route choice in flying honeybees: evidence for individual handedness. PLoS ONE 12, e0184343.

Osborne, J. L., Martin, A. P., Carreck, N. L., Swain, J. L., Knight, M. E., Goulson, D., Hale, R. J. and Sanderson, R. A. (2008). Bumblebee flight distances in relation to the forage landscape. J. Anim. Ecol. 77, 406-415.

Philippides, A., de Ibarra, N. H., Riabinina, O. and Collett, T. S. (2013) Bumblebee calligraphy: the design and control of flight motifs in the learning and return flights of Bombus terrestris. J. Exp. Biol. 216, 1093-1104.

Ravi, S., Crall, J. D., Fisher, A. and Combes, S. A. (2013). Rolling with the flow: bumblebees flying in unsteady wakes. J. Exp. Biol. 216, 4299-4309.

Ravi, S., Kolomenskiy, D., Engels, T., Schneider, K., Wang, C., Sesterhenn, J. and Liu, H. (2016). Bumblebees minimize control challenges by combining active and passive modes in unsteady winds. Sci. Rep. 6, 35043.

Riabinina, O., de Ibarra, N. H., Philippides, A. and Collett, T. S. (2014). Head movements and the optic flow generated during the learning flights of bumblebees. J. Exp. Biol. 217, 2633-2642.

Schiffner, I., Vo, H. D., Bhagavatula, P. S. and Srinivasan, M. V. (2014). Minding the gap: in-flight body awareness in birds. Front. Zool. 11, 64.

Serres, J. R. and Ruffier, F. (2017). Optic flow-based collision-free strategies: from insects to robots. Arthropod Struct. Dev. 46, 703-717.

Serres, J., Dray, D., Ruffier, F. and Franceschini, N. (2008). A vision-based autopilot for a miniature air vehicle: joint speed control and lateral obstacle avoidance. Aut. Robot 25, 103-122.

Shyy, W., Kang, C.-K., Chirarattananon, P., Ravi, S. and Liu, H. (2016) Aerodynamics, sensing and control of insect-scale flapping-wing flight Proc. R. Soc. A Math. Phys. Eng. Sci. 472

Sobel, E. C. (1990). The locust's use of motion parallax to measure distance. $J$ Comp Physiol A 167, 579-588.

Srinivasan, M. V. (2010). Honey bees as a model for vision, perception, and cognition. Annu. Rev. Entomol. 55, 267-284.

Srinivasan, M. V. (2015). Where paths meet and cross: navigation by path integration in the desert ant and the honeybee. J. Comp. Physiol. A 201, 533-546.

Srinivasan, M. V. and Zhang, S. W. (1997). Visual control of honeybee flight. EXS 84, 95-113.

Srinivasan, M. V., Lehrer, M. and Horridge, G. A. (1990). Visual figure- ground discrimination in the honeybee: the role of motion parallax at boundaries Proc. R. Soc. Lond. B. 238, 331-350.

Taylor, G. K. (2001). Mechanics and aerodynamics of insect flight control. Biol. Rev. Camb. Philos. Soc. 76, 449-471.

van Breugel, F., Morgansen, K. and Dickinson, M. H. (2014). Monocular distance estimation from optic flow during active landing maneuvers. Bioinspir. Biomim. 9, 25002

Wallace, G. K. (1959). Visual scanning in the desert locust Schistocerca gregaria Forskgd. J. Exp. Biol. 36, 512-525.

Zeil, J. (1996). The control of optic flow during learning flights. J. Comp. Physiol. A Sensory Neural Behav. Physiol. 180, 25-37.

Zhang, S. W. and Srinivasan, M. V. (1994). Prior experience enhances pattern discrimination in insect vision. Nature (Lond) $\mathbf{3 6 8}, 330-332$ 